\title{
Caenorhabditis elegans UNC-103 ERG-Like Potassium Channel Regulates Contractile Behaviors of Sex Muscles in Males before and during Mating
}

\author{
L. Rene Garcia ${ }^{1}$ and Paul W. Sternberg ${ }^{2}$ \\ ${ }^{1}$ Department of Biology, Texas A\&M University, College Station, Texas 77843-3258, and ${ }^{2}$ Howard Hughes Medical Institute and Division of Biology, \\ California Institute of Technology, Pasadena, California 91125
}

\begin{abstract}
During mating behavior the Caenorhabditis elegans male must regulate periodic and prolonged protractor muscle contractions to insert his copulatory spicules into his mate. The protractors undergo periodic contractions to allow the spicules to reattempt insertion if a previous thrust failed to breach the vulva. When the spicule tips penetrate the vulva, the protractors undergo prolonged contraction to keep the spicules inside the hermaphrodite until sperm transfer is complete. To understand how these contractions are regulated, we isolated EMS-induced mutations that cause males to execute prolonged contraction inappropriately. Loss-of-function mutations in the unc-103 ERG-like $\mathrm{K}^{+}$channel gene cause the protractor muscles to contract in the absence of mating stimulation. unc-103-induced spicule protraction can be suppressed by killing the SPC motor neurons and the anal depressor muscle: cells that directly contact the protractors. Also, reduction in acetylcholine suppresses unc-103-induced protraction, suggesting that UNC-103 keeps cholinergic neurons from stimulating the protractors before mating behavior. UNC-103 also regulates the timing of spicule protraction during mating behavior. unc-103 males that do not display mating-independent spicule protraction show abnormal spicule insertion behavior during sex. In contrast to wild-type males, unc-103 mutants execute prolonged contractions spontaneously within sequences of periodic protractor contractions. The premature prolonged contractions cause the spicules to extend from the male tail before the spicule tips penetrate the vulva. These observations demonstrate that $u n c-103$ controls various aspects of spicule function.
\end{abstract}

Key words: Caenorhabditis elegans; mating behavior; unc-103; ERG; ${ }^{+}$channel; L-type voltage-gated calcium channels

\section{Introduction}

Analysis of Drosophila behavioral mutants led to the first in vivo correlation among $\mathrm{K}^{+}$channel genes such as shaker (Kamb et al., 1987; Temple et al., 1987; Pongs et al., 1988), slowpoke (Atkinson et al., 1991), ether-a-go-go (Kaplan and Trout, 1969; Warmke et al., 1991), and seizure/erg (Titus et al., 1997; Wang et al., 1997) and their roles in specific motor outputs (Papazian et al., 1988). Mutations that affect these $\mathrm{K}^{+}$channels also perturb more complex behaviors such as habituation of escape response (Engel and Wu, 1998), courtship behavior (Griffith et al., 1994), and olfaction (Cowan and Siegel, 1986).

In Caenorhabditis elegans genetic screens also have identified $\mathrm{K}^{+}$channel genes that regulate different behaviors. Gain-offunction mutations in the $\mathrm{K}^{+}$channel genes like unc-103 (the homolog of seizure/erg) (Reiner et al., 1999; J. H. Thomas, personal communication), egl-36 (the homolog of shaw) (Elkes et al., 1997; Johnstone et al., 1997), egl-2 (the homolog of ether-a-go-go) (Weinshenker et al., 1999), twk-18 (two-P domain $\mathrm{K}^{+}$channel)

\footnotetext{
Received Nov. 13, 2002; revised Jan. 9, 2003; accepted Jan. 13, 2003.

The Howard Hughes Medical Institute with which P.W.S. is an investigator and L.R.G. was an associate supported this research. L.R.G. was supported by a Department of Health and Human Services National Research Service Award (GM18857). Strains were provided by the Caenorhabditis Genetics Center, which is supported by National Institutes of Health National Center for Research Resources. We thank David Reiner and James Thomas for communicating to us the molecular lesions in unc-103(e1597gf) and unc-103(n1213). We also thank Karin Doerr for writing software used in this work and Gary Schindelman for comments on this manuscript.

Correspondence should be addressed to L. Rene Garcia, Department of Biology, Texas A\&M University, 3258 TAMU, College Station, TX 77843-3258. E-mail: rgarcia@mail.bio.tamu.edu. Copyright $\odot 2003$ Society for Neuroscience $\quad 0270-6474 / 03 / 232696-10 \$ 15.00 / 0$
}

(Kunkel et al., 2000), and exp-2 (a novel $\mathrm{K}_{\mathrm{v}}$ channel) (Davis et al., 1999) reduce locomotion, defecation, and egg-laying behaviors (Trent et al., 1983; Park and Horvitz, 1986; Thomas, 1990; Reiner et al., 1995). Loss-of-function mutations in slo-1 (calciumactivated, voltage-gated $\mathrm{K}^{+}$channel) suppress the general lethargy of unc-64 (syntaxin) mutants (Wang et al., 2001).

C. elegans male mating behavior is a complex behavior that requires the coordination of different motor outputs (Ward and Carrel, 1979; Loer and Kenyon, 1993; Barker, 1994; Liu and Sternberg, 1995; Barr and Sternberg, 1999; Garcia et al., 2001; Simon and Sternberg, 2002). To sire progeny, the male must insert his copulatory spicules into his mate; this process requires the regulation of two types of spicule protractor muscle contractions. During spicule insertion behavior the PCB and PCC postcloacal sensory neurons signal the protractor muscles to contract and relax at a frequency of $7-9 \mathrm{~Hz}$, causing the spicules to prod the vulval slit repeatedly. When the spicules penetrate the vulva, the SPC motor neurons signal periodic contractions to cease and cause the protractor muscles to remain contracted until sperm transfer is completed (Garcia et al., 2001).

To understand how distinct motor outputs are regulated, we isolated mutations that induce spontaneous spicule protraction. We find that mutations in $u n c-103$, the worm homolog of Drosophila seizure/erg-encoded and the human $h$-erg-encoded delayed inward rectifying voltage-gated $\mathrm{K}^{+}$channel, affect the spicule insertion step (Sanguinetti et al., 1995; Trudeau et al., 1995; Titus et al., 1997; Wang et al., 1997; Reiner et al., 1999; J. H. Thomas, personal communication). 
$h$-erg encodes the cardiac $I_{\mathrm{Kr}} \mathrm{K}^{+}$channel that facilitates cardiac rhythms (Sanguinetti et al., 1995). HERG channels display fast inactivation that reduce potassium efflux during depolarization and display rapid recovery during repolarization (Trudeau et al., 1995). In humans and flies, mutations in erg have profound effects on the organism. Individuals that contain $h$-erg mutations can suffer from lethal heart arrhythmias (Curran et al., 1995), and flies with sei/erg mutations display temperature-induced seizures (Titus et al., 1997; Wang et al., 1997). In contrast, we find that in C. elegans males UNC-103 has a specific role in mating behavior, and it regulates sex muscle contractions before and during copulation.

\section{Materials and Methods}

Strains. All strains used in this study contain him-5(e1490) on LGV (Hodgkin et al., 1979). We maintained the strains by using standard culture conditions (Brenner, 1974). Our wild-type reference strain, PS1395, contains plg-1(e2001) on LGIII. This allele allows males to secrete a gelatinous plug over the vulva after sperm transfer (Hodgkin and Doniach, 1997). We also used the following alleles in this study: unc38 (sy576) (Garcia et al., 2001) and unc-13(e51) (Brenner, 1974) on LGI; daf-2(e1370) (Riddle, 1977), unc-93(e1500) (Greenwald and Horvitz, 1980), unc-79(e1068) (Hodgkin, 1983), unc-103(e1597gf) (Hodgkin, 1983), unc-103(n1213) (Park and Horvitz, 1986), pal-1(e2091) (Waring and Kenyon, 1990), dpy-17(e164) (Brenner, 1974), pha-1(e2123) (Schnabel and Schnabel, 1990), unc-49(e382) (Brenner, 1974), and unc64(e246) (Brenner, 1974) on LGIII; unc-31(e169) (Brenner, 1974) and egl-19(n582) (Trent et al., 1983) on LGIV; unc-68(r1158) (Maryon et al., 1996) on LGV; cat-1(e1111) (Sulston et al., 1975) and unc-2(e55) (Brenner, 1974) on LGX. For single nucleotide polymorphism (SNP) mapping we used the Hawaiian CB4856 strain as the source of the polymorphisms (Wicks et al., 2001).

Isolation of sy557 and scoring mating-independent spicule protraction. To isolate sy557, we first mutagenized PS1395 hermaphrodites by using ethyl methanesulfonate (EMS) (Brenner, 1974). After the EMSmutagenized hermaphrodites produced the F1 generation, we put single F1 hermaphrodites on Petri plates and allowed them to produce the F2 generation. We identified plates in which F2 males and hermaphrodites had general wild-type behavior and in which approximately one-fourth of the males had protruding spicules. We then isolated a homozygous line by picking individual sibling hermaphrodites to separate plates. We kept plates in which the majority of progeny males had protruding spicules. From 10,000 mutagenized gametes we isolated sy557, sy558, sy559, and sy574.

To isolate sy670, sy673, and sy674, we followed the procedure of Park and Horvitz (1986). We EMS-mutagenized unc-103(e1597); him-5(e1490); in the F2 generation we isolated hermaphrodites with wild-type behavior. We then scored how many progeny males spontaneously protracted their spicules.

sy557 animals were out-crossed six times to N2 strains. We outcrossed sy670, sy673, and sy674 animals to non-mutagenized unc103(e1597) two times before we assayed their behavior. To score matingindependent spicule protraction, we first removed L4 males away from hermaphrodites, putting 20-30 males together on a Petri plate. Then 24 hr later we used a Wild M5A microscope to count how many males had protruding spicules. We continued to score the males for $2 \mathrm{~d}$ additionally; however, permanent spicule protraction generally occurred within the first $24 \mathrm{hr}$ of adulthood. Mating-independent spicule protraction is not dependent on population density. The frequency of spicule protraction did not change if individuals were isolated or 150 males were kept together (data not shown). However, if too few males were kept together, the males crawled off the agar and dried on the side of the Petri plate; if too many males were kept together, the males would aggregate, damage the agar surface, and burrow.

Mating efficiency. We adapted the mating efficiency assay from Hodgkin (1983). To measure mating efficiency, we placed six L4 males with six L4 unc-64(e246) hermaphrodites on a standard Escherichia coli OP50-seeded NG plate. We considered each plate as a single mating trial.
Then 3-4 d later we counted the total number of paralyzed and nonparalyzed cross progeny. Mating efficiencies were calculated as the percentage of cross progeny divided by the number of total progeny.

Mapping of sy557. We used standard mapping procedures to threefactor map sy557 (Brenner, 1974). In all cases we selected recombinants on the basis of visible hermaphrodite markers and then determined whether recombinant males displayed the sy557 phenotype. The results from the three-factor mapping experiments are as follows: from unc-93 $d p y-17 /$ sy557 heterozygotes, 9 of 13 Dpy, non-Unc recombinants segregated sy557; from daf-2 unc-79/sy557 heterozygotes, 6 of 6 Daf, non-Unc and 0 of 3 Unc, non-Daf recombinants segregated sy557; from $u n c-103$ (e1597) $d p y$-17/sy557 heterozygotes, 5 of 5 Dpy, nonUnc and 0 of $8 \mathrm{Unc}$, non-Dpy recombinants segregated sy557; from unc-79 pal-1/sy557 recombinants, 0 of $35 \mathrm{Unc}$, non-Pal and 1 of $1 \mathrm{Pal}$, non-Unc recombinants segregated sy557. These data suggested that sy557 mapped near unc-79 and unc-103.

We also used standard SNP mapping procedure to three-factor map sy557 in relation to SNPs that are located between unc-93 and pal-1 (Wicks et al., 2001). The locations of the SNPs in the CB4856 are detailed at http://genome.wustl.edu/projects/celegans. To analyze the SNPs, we used the following primer pairs to amplify the genomic regions of the recombinants: pkP3096 (5'-CCAACTGTCGACGATAGAGATG-3' and 5'-AATTCCGTGTAGCGAATCG-3'); pkP3097 (5'-TTTCAAGACAAGGCAAGTATCC-3' and 5' ${ }^{\prime}$-TTTGATCAATCAGTGGGTCTTG-3'); snp C30D11.4 (5' ${ }^{\prime}$-TGCTCCACGGATCGACTTGAGAGTGCCGTTGG- $3^{\prime}$ and $5^{\prime}$-CGCTCAACTTCACAATTTCGTAGATGTATATC-3'); snp R10E4.1 (5'-GTCGGCGGCATCGAGTGAAGCC-3' and 5'-GTACCATTATTCGAGCTCAAGGTC-3'); snp C28A5.2 (5'-TTGCATACGTGCCTTTAAGAG-3' and $5^{\prime}$-CAGAAAGACAGAAAAGGGTCG-3'). We analyzed the SNPs either by DNA sequencing or restriction analysis.

The results from the SNP mapping experiments are as follows: from daf-2 sy557dpy-17(N2)/+ (CB4856) heterozygotes, 1 of 16 daf- 2 sy557+ recombinants contained snp C28A5.2 from CB4856 and 4 of 16 recombinants contained both snp C28A5.2 and snp R10E4.1 from CB4856; from daf-2 sy557 dpy-17(N2)/+ (CB4856) heterozygotes, 23 of 24 daf-2++ recombinants contained the SNPs pkP3096, pkP3097, snp C30D11.4, snp R10E4.1, and snp C28A5.2 from CB4856, and 1 of 24 recombinants contained snp C30D11.4, snp R10E4.1, and snp C28A5.2 from CB4856. These data suggested that sy557 mapped in a region between snp C30D11.4 and snp R10E4.1

Pharmacology. We dissolved arecoline (purchased from SigmaAldrich, St. Louis, MO) in water to make a stock solution of $100 \mathrm{~mm}$. We then serially diluted the stock solution in water as needed. We added $1 \mathrm{ml}$ of the drug to a Pyrex round-bottom, three-well titer dish. Then 5-10 $<24 \mathrm{hr}$ adult virgin males were transferred to the drug bath. We observed the males for 5 min with a Wild M5A microscope. The males were considered responsive to the drug if they kept their spicules protracted for $\geq 10 \mathrm{sec}$.

Laser ablations. We used standard laser ablation protocols to kill cells in the male tail (Bargmann and Avery, 1995). To ablate neurons, we operated on L4 males that were at a stage in development after the tail ventral hypodermis completely retracted. To ablate the precursor cells P9.p, P10.p, P11.p, F(1/r), and U(1/r), we operated on early L3 males. To rule out any effects that the anesthetic might have on sy557-induced behavior, we kept nonoperated cohorts on agar pads soaked with anesthetics similar to the operated males.

unc-103 reporter construction. We PCR-amplified an $8.2 \mathrm{~kb}$ fragment of DNA that contains $5 \mathrm{~kb}$ upstream of the unc-103 start codon and includes part of the seventh exon of unc-103. We used N2 genomic DNA as the template for the PCR and the primers $5^{\prime}$-GGGCACGCCTGCCTAAGGGATGCCTTAGCTCGGCC-3' and 5'-CTGGTGAAA TCGGATAAATTCTCTG-3' to amplify the DNA fragment. We then blunt end-ligated the PCR fragment into the SmaI site of pPD95.79 (plasmid courtesy of A. Fire, Carnegie Institute of Washington, Baltimore, MD) to make the plasmid pR48. Green fluorescent protein (GFP) is fused $\sim 20$ amino acids after the $S 6$ transmembrane domain. pR48 $(30 \mathrm{ng} / \mu \mathrm{l})$ and the wild-type $p h a-$ 1 -containing plasmid pBX-1 $(100 \mathrm{ng} / \mu \mathrm{l})$ were injected into the germline of pha-1; him-5 hermaphrodites (Mello et al., 1991; Granato et al., 1994). 
Twenty mid-L4 males from two transgenic lines were analyzed for unc103 expression.

We noted that the fusion protein aggregates mostly on the cytoplasmic membrane of neuronal processes and in many, but not all, cases of cell bodies; however, we detected no deleterious effect of the fusion construct on the behavior of the transgenic animals. In larval hermaphrodites and males we were able to identify some of the cells that had cell body localization of UNC-103::GFP; many neurons express the construct, but because of variability in cell position and cellular localization of the fusion protein in processes, our list of unc-103-expressing cells is far from complete. In the head region ALA, ADL, ASK, AVH, AVJ, AIN, AVA, ASJ, SMDD, SIA, ADE, and AVD express the construct. In the tail region PHA, DVC, ALN, and PVP express the construct. The unc-103 fusion protein accumulates extensively in the processes of many ventral cord neurons; however, we were able to identify only some cells in the AS class of excitatory neurons. In the adult hermaphrodite HSN expresses the unc-103::gfp construct.

Injections of $\mathrm{pR} 48$ at $\geq 50 \mathrm{ng} / \mu \mathrm{l}$ were lethal. Some F1 transgenic animals were obtained with $50 \mathrm{ng} / \mu \mathrm{l} \mathrm{pR} 48$; however, they did not transmit the extrachromosomal array to subsequent generations. These exceptional F1 animals showed mosaic expression of GFP not only in neurons but also in body wall and pharyngeal muscles (our unpublished observation). Therefore, we cannot rule out that unc-103 may be expressed in more cell types than neurons, but neurons might tolerate the expression of the fusion construct better than muscles.

Mating observation. We observed mating behavior with a compound microscope fit with a $40 \times$ objective. Mating behavior was recorded with an MTI CCD72 black-and-white video camera and a Panasonic AG-6740 time-lapse Super VHS videocassette recorder. We placed five 15-24 hr unc-38(sy576);egl-19(n582) adult hermaphrodites and one male on a 5 -mm-diameter OP50 lawn made on a $1 \mathrm{~cm}$ block of agar. We then placed the agar block on a microscope slide for direct viewing. When the male tail touched the vulva, we started the video recording. Males have difficulty inserting their spicules into young hermaphrodites and will prod the vulva continuously for $10 \mathrm{~min}$ or longer. This allowed us to make long recordings of this behavior. To quantify the rate of spicule movements for wild type and unc-103 null, we slowed the recordings to $1 / 7$ the original speed and manually counted the downstrokes of the spicules.

\section{Results \\ unc-103 males protract their copulatory spicules independently of mating stimulation}

The C. elegans male contains two copulatory spicules. Each spicule is attached to two protractor muscles (Fig. $1 A$ ). When these muscles contract, the attached spicules extrude from the male tail. To understand how specific motor behaviors are regulated during copulation, we screened for mutations that uncouple the spicule insertion step from other mating-specific motor and sensory behaviors. From a screen of 10,000 EMS-mutagenized gametes we isolated the sy557 allele. Wild-type males keep their spicules extended from their tails only during mating. In contrast, sy557 males spontaneously protract their spicules in the absence of mating stimulation.

Initially after the L4 molt sy557 adult males behave normally, but within 1-5 hr they spontaneously protract their spicules (Fig. $1 B-D)$. Occasionally, mating-independent protraction is coupled with a dorsal or a ventral tail curl. Affected males sometimes can retract their spicules into their tails; however, their spicules will continue to protract inappropriately. Eventually, the spicules remain permanently protracted.

This mutant phenotype is similar to the protruding spicule phenotype of males that contain the gain-of-function egl19(n2368gf) allele (Lee et al., 1997; Garcia et al., 2001). egl-19 encodes the C. elegans homolog of the L-type voltage-gated calcium channel $\alpha 1$ subunit (Lee et al., 1997). In egl-19( $g f)$ males
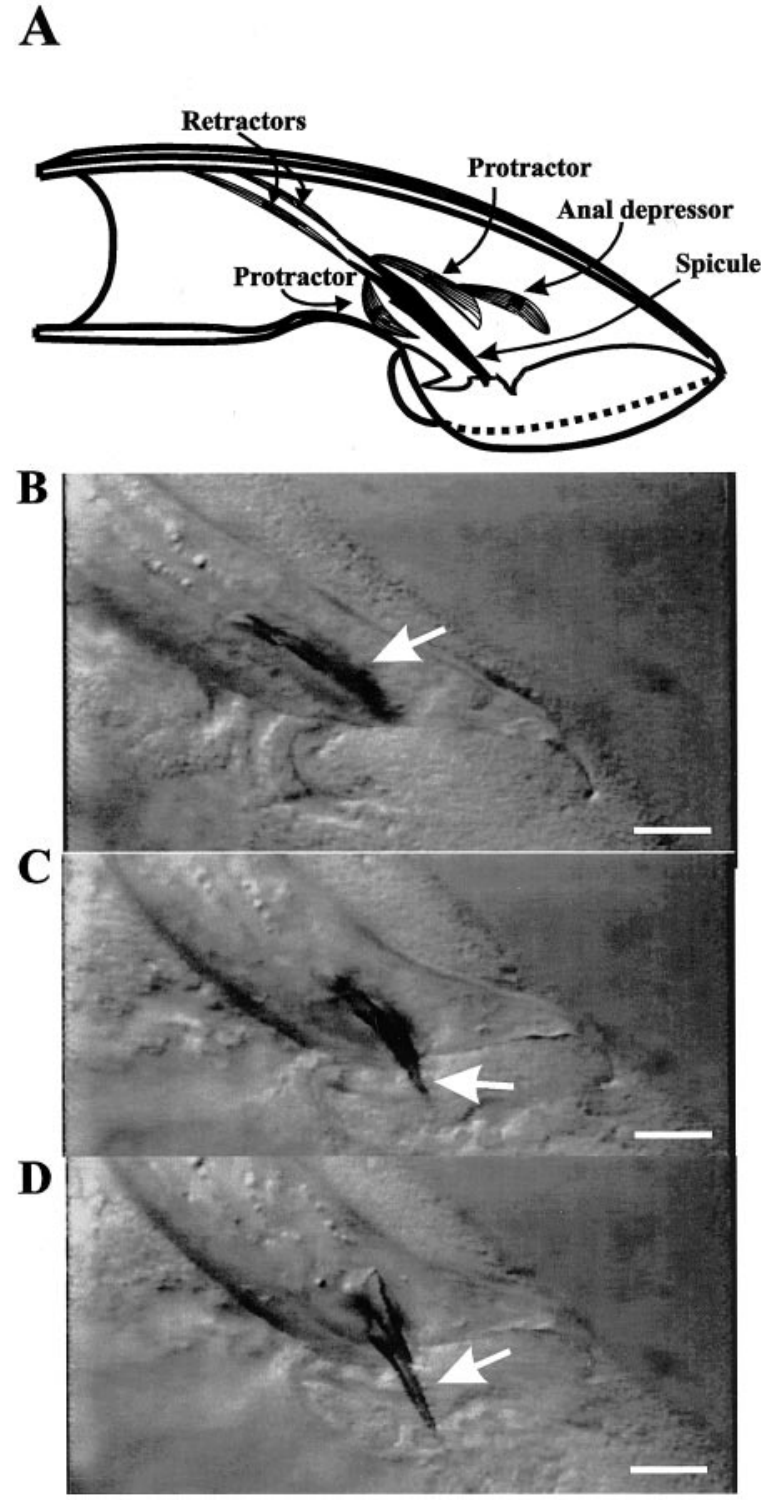

Figure 1. Mating-independent spicule protraction displayed by a sy557 mutant male. $A$ depicts a diagram of the sex muscles that are associated with the spicules (right lateral view of the male tail). The drawing was adapted from Sulston et al. (1980). The spicule is shown in black. The dorsal and ventral protractor and retractor muscles, represented as striated cells, are attached to the body wall and the base of the spicule. The anal depressor muscle is attached to the body wall and contacts the dorsal protractor. $B-D$ depict individual frames taken from a video recording of a $<24 \mathrm{hr}$ virgin sy 557 adult male. The arrow points to his copulatory spicules (lateral view). In $B$, the arrow points to copulatory spicules that are inside his tail. In C, the arrow points to the copulatory spicule as it starts to protrude from the tail. $\ln D$, the spicule is protracted completely. In all panels his anterior is to the left, and his dorsal side is to the top. Scale bar, $20 \mu \mathrm{m}$.

excessive inappropriate channel activity not only may activate protractor contraction prematurely but also subsequently may damage the spicule muscle cells so that the spicules no longer can be retracted. In affected sy557 males a similar type of defect also might be occurring.

In a population of virgin males kept isolated from hermaphrodites, $\sim 60 \%$ of the males will protract their spicules permanently within $24 \mathrm{hr}$ after they emerge from their L4 cuticle (Table 1). Once the spicules remain protracted, the sy557 male is incapable of siring progeny. Exceptional sy557 males that do not display mating-independent spicule protraction can sire progeny, 
Table 1. unc-103 mutants display mating-independent spicule protraction

\begin{tabular}{lcc}
\hline Genotype & Percentage of & \\
\hline Wild type & protracted males $^{a}$ & $n^{b}$ \\
unc-103(sy557) H165N, W244R & 0 & 100 \\
$+/$ unc-103(sy557) & 66 & 454 \\
unc-103(e1597gf) & 20 & 487 \\
$+/$ unc-103(e1597) & 0 & 80 \\
unc-103(sy673, e1597) W85opal & 0 & 62 \\
unc-103(sy670, e1597) G306E & 20 & 312 \\
unc-103(e1597, sy674) P4265 & 37 & 81 \\
unc-103(n1213) null & 7 & 46 \\
$+/$ lunc-103(n1213) null & 29 & 324 \\
unc-103(sy557)/unc-103(n1213) & 0 & 64 \\
unc-103(sy673, e1597)/unc-103(n1213) & 40 & 32 \\
unc-103(sy670, e1597)/unc-103(n1213) & 13 & 78 \\
unc-103(e1597, sy674)/unc-103(n1213) & 17 & 29 \\
\hline
\end{tabular}

${ }^{a}$ Males whose spicules remain permanently protracted.

${ }^{b}$ Number of males assayed.

albeit at an efficiency less than wild-type males (wild-type mating efficiency: $0.74 \pm 0.11$, mean $\pm \mathrm{SD}, n=3$ trials; sy557 mating efficiency: $0.29 \pm 0.12, n=2$ trials).

sy557 males and hermaphrodites do not show any gross abnormalities in other behaviors such as locomotion, pharyngeal pumping behavior, defecation, or chemotaxis. However, sy557 hermaphrodites sometimes lay eggs at a slightly higher rate than wild type (our unpublished observation).

We mapped sy557 to LGIII between $d a f-2$ and $d p y$-17 (see Materials and Methods). Using single nucleotide polymorphisms as mapping markers (Wicks et al., 2001), we narrowed sy557 to a $140 \mathrm{~kb}$ interval between the polymorphisms snp C30D11.4 and snp R10E4.1. We noted that unc-103, the C. elegans homolog of Drosophila seizure/erg and human h-erg (Titus et al., 1997; Reiner et al., 1999; J. H. Thomas, personal communication), was in this interval. Loss-of-function mutations in the Drosophila $\mathrm{K}^{+}$channel gene cause general fly hyperactivity and spontaneous seizures (Jackson et al., 1984, 1985; Kasbekar et al., 1987; O’Dowd and Aldrich, 1988; Elkins and Ganetzky, 1990). The phenotypic similarity of spontaneous C. elegans male protractor contractions and spontaneous seizures in Drosophila suggested that sy557 might affect the $\mathrm{K}^{+}$channel gene in C. elegans.

We sequenced the predicted coding regions of unc-103 in our reference wild-type strain and in the sy557 mutant and found that the sy557 mutant contains two missense mutations in unc-103. The mutations change a histidine at amino acid 165 to an asparagine near the S3 transmembrane region and a tryptophan at position 244 to an arginine in the S5 region. Both of these substitutions are in amino acids that are conserved in the human, fly, and worm protein (Fig. 2).

\section{Reduction of unc-103 causes spontaneous spicule protractor contraction}

To determine how the two missense mutations in unc-103(sy557) cause mating-independent spicule protraction, we compared the phenotype induced by unc-103(sy557) with the phenotypes of loss-of-function, gain-of-function, and null alleles of unc-103. unc-103(sy557) is semidominant because heterozygous males protract their spicules (Table 1). To determine whether semidominance was attributable to sy557 conferring enhanced activity to UNC-103, we first compared unc-103(sy557) with the canonical gain-of-function $u n c-103(e 1597 g f)$ allele. unc-103 was defined first by the dominant gain-of-function mutations e1597 and $n 500$ (Hodgkin, 1983; Park and Horvitz, 1986). Animals containing unc-103(e1597gf) are extremely lethargic and egg-laying defective in both the homozygous and heterozygous state (Hodgkin, 1983; Park and Horvitz, 1986; Reiner et al., 1995). None of these behavioral defects is seen in unc-103(sy557) animals. An additional difference is that $u n c-103(e 1597 g f)$ males do not protract their spicules spontaneously (Table 1). Thus the basis for the semidominance of unc-103(sy557) is probably not the same as for $u n c-103(e 1597 g f)$.

Work by others has shown that suppression of unc-103 (e1597gf)-induced lethargy and defective egg-laying behaviors can be caused by intragenic mutations that reduce gene function (Park and Horvitz, 1986). To determine whether unc-103(sy557) caused a reduction in UNC-103 activity, we isolated additional intragenic revertants of $u n c-103(e 1597 g f)$ and asked whether intragenic mutations that eliminate the 1597 phenotype also can induce spicule protraction. We first EMS-mutagenized unc103(e1597gf) animals and then selected F2 hermaphrodites that had wild-type locomotion and egg-laying behavior. We then assayed their male progeny for spontaneous spicule protraction. We isolated three intragenic revertants of unc-103(e1597gf): unc103(sy673, e1597gf) W85opal, unc-103(sy670, e1597gf) G306E, and unc-103(e1597gf, sy674) P426S. sy673 creates a stop codon that truncates the gain-of-function protein after the first 85 amino acids, which is a few hundred amino acids upstream of the e1597 lesion (J. H. Thomas, personal communication); sy670 changes the conserved glycine at amino acid position 306 to a glutamic acid in the ion selectivity filter of the pore region (Doyle et al., 1998); and sy674 changes a proline at amino acid position 426 to a serine, which is downstream of the e1597 lesion (J. H. Thomas, personal communication) (Fig. 2).

In all, 20 and $37 \%$ of males containing unc-103(sy673, e1597gf) W85opal and unc-103(sy670, e1597gf) G306E, respectively, displayed seizures of their protractor muscles; in contrast, only $7 \%$ of unc-103(e1597gf, sy674) P426S showed the phenotype. We believe that both the W85opal and G306E lesions reduce $\mathrm{K}^{+}$channel function of the gain-of-function protein. The stop mutation truncates the gain-of-function protein within the first transmembrane region, and others have demonstrated that mutating the equivalent glycine in the ion selectivity filter of the human HERG channel disrupts ion conduction (Sanguinetti et al., 1996). We also assayed the effects that an unc-103 null mutation had on spontaneous protractor contraction. unc-103(n1213) was isolated originally as a revertant of $u n c-103(\mathrm{e} 1597 \mathrm{~g} f$ ) (Park and Horvitz, 1986) and is a deletion of unc-103 (Reiner et al., 1999; J. H. Thomas, personal communication). Similar to unc-103(sy673, e1597gf) W85opal and unc-103(sy670, e1597gf) G306E males, $29 \%$ of unc-103(n1213) null males showed spontaneous protractor muscle contraction (Table 1). Thus we conclude that reduction of UNC-103 activity results in mating-independent spicule protraction. The unc-103(e1597gf, sy674) P426S mutation had a small effect on activating premature spicule protraction, suggesting that the e1597gf, sy674 channel still might have some functional activity. Because unc-103(e1597gf, sy674) animals show locomotion and egg-laying behavior similar to wild type, the P426S change might counteract the effects of the e 1597 mutation so that some level of proper channel function is restored.

Although loss-of-function and null mutations in unc-103 caused mating-independent spicule protraction, the percentages of males showing the defect were lower compared with unc103(sy557) H165N, W244R males. To test whether UNC-103 (H165N, W244R) also might interfere with wild-type UNC-103 function, we analyzed its genetic interaction with the gain-of- 
function unc-103 allele. Arecoline, an acetylcholine agonist, can induce the spicule protractor muscles to contract artificially (Garcia et al., 2001). The unc-103(e1597gf) mutation reduces the effectiveness of the agonist to induce muscle contraction (Fig. $3 A$ ). At a concentration in which $\sim 90 \%$ of wild-type males respond to the drug, homozygous unc-103(e1597gf) males are insensitive, and $30 \%$ of $u n c-103(e 1597 g f) /+$ males and $38 \%$ of $u n c-103(e 1597 g f) /$ null males respond (Fig. $3 B$ ). We put unc$103(e 1597 g f)$ in trans to the other $u n c-103$ alleles to determine whether they could interfere with the gain-of-function mutant channel and consequently restore drug sensitivity. When we made comparisons to unc-103(e1597gf)/null, we found that putting unc-103(sy673, e1597gf) W85opal or unc-103 (e1597gf, sy674) P426S in trans to $u n c-103(e 1597 g f)$ reduces sensitivity to arecoline. This suggests that for sy673 translational read-through might be occurring at the opal stop codon so that some level of full-length e1597 protein is made; for sy674 the P426S change probably does not suppress the 1597 lesion completely. In contrast, unc-103(sy670, e1597gf) G306E can interfere partially with $u n c-103(e 1597 g f)$; this is not surprising because others have shown that mutating the glycine at that position in the ion selectivity filter confers dominantnegative properties to the protein (Sanguinetti et al., 1996). Additionally, we found that unc-103(sy557) H165N, W244R completely interfered with $u n c-103(e 1597 g f)$ and restored drug sensitivity to wild-type level, thus suggesting that unc-103(sy557) also might act as a dominant-negative protein. However, because mating-independent spicule protraction occurred more frequently in unc-103(sy557) than the null, the UNC-103 (H165N, W244R) protein must have, additionally, properties that cause it to interfere with the regulation of spicule protraction behavior.

The SPC motor neurons and the anal depressor muscle facilitate $u n c$-103(sy557)-induced spicule protraction

The SPC motor neurons and the anal depressor muscle are attached directly to the protractor muscles (Fig. 4A) (Sulston et al., 1980). During mating the SPC motor neurons are essential for triggering prolonged contraction; in contrast, the anal depressor muscle is nonessential for mating behavior under standard conditions (Garcia et al., 2001). We ablated the SPC motor neurons and the anal depressor muscle to determine whether these cells were required for $u n c-103$ (sy557)-mediated spicule protraction. Ablating either cell type separately did not affect the mutant phenotype; however, when both cells were killed, the spicules did not protract spontaneously (Table 2). This observation suggests that unc-103(sy557) does not act solely in the protractor muscles to cause spontaneous spicule protraction. We ablated other neurons in the vicinity of the protractor and anal depressor muscles (Sulston et al., 1980), but unlike the SPC-anal depressor double ablation, killing combinations of other neurons did not eliminate mutation-induced protraction (Table 2 ).

Because unc-103(sy557) does not act solely in the protractor muscles, we fused GFP to $u n c-103$ sequences to determine which of the cells associated with the spicules express the gene. Our
unc-103::GFP construct expressed broadly in the head and ventral cord neurons in both males and hermaphrodites (see Materials and Methods). In the male tail the SPC motor neurons and the PCB postcloacal sensory neurons expressed the construct (Fig. $4 B$ ). The expression in SPC is consistent with those neurons contributing to unc-103(sy557)-induced spicule protraction. Irregular expression was seen in the PCA postcloacal sensory neurons; in other neurons that are associated with spicules, such as the PCC postcloacal sensory neurons and the SPD and SPV spicule sensory neurons, the fusion protein was not expressed.

The unc-103(sy557) phenotype requires acetylcholine production and L-type voltage-gated calcium channels To determine which molecular components are acting inappropriately when $u n c-103$ is compromised, we surveyed mutations that affect general synaptic transmission and muscle contraction for suppression of the unc-103(sy557) phenotype. We found that mutations in cat-1 [vesicular monoamine transporter (Duerr et al., 1999)], unc-49 [GABA receptor (Bamber et al., 1999)], unc-38 [nicotinic acetylcholine receptor subunit (Fleming et al., 1997)], unc-64 [syntaxin 1A (Ogawa et al., 1998; Saifee et al., 1998)], and unc-2 [ $\alpha 1$ voltage-gated calcium channel subunit (Schafer and Kenyon, 1995)] did not reduce $u n c-103($ sy557)-induced protraction. However, mutations in unc-31 [neurosecretory calciumbinding protein (Ann et al., 1997)], unc-13 [DAG-binding synaptic transmission component (Ahmed et al., 1992)], and unc-68 [ryanodine receptor calcium channel (Maryon et al., 1996)] had a very small to moderate effect on reducing spicule protraction. In contrast, mutations in cha-1 [choline acetyltransferase (Alfonso et al., 1994)] and egl-19 [L-type $\alpha 1$ voltage-gated calcium channel subunit (Lee et al., 1997)] suppressed the unc-103(sy557) phenotype (Table 3 ). The suppression by cha-1 and egl-19 suggests that spontaneous protractor contraction requires L-type voltagegated calcium channels and acetylcholine (ACh) production. This suppression was expected because EGL-19 is required for prolonged protractor contraction during mating behavior, and 
$\mathbf{A}$
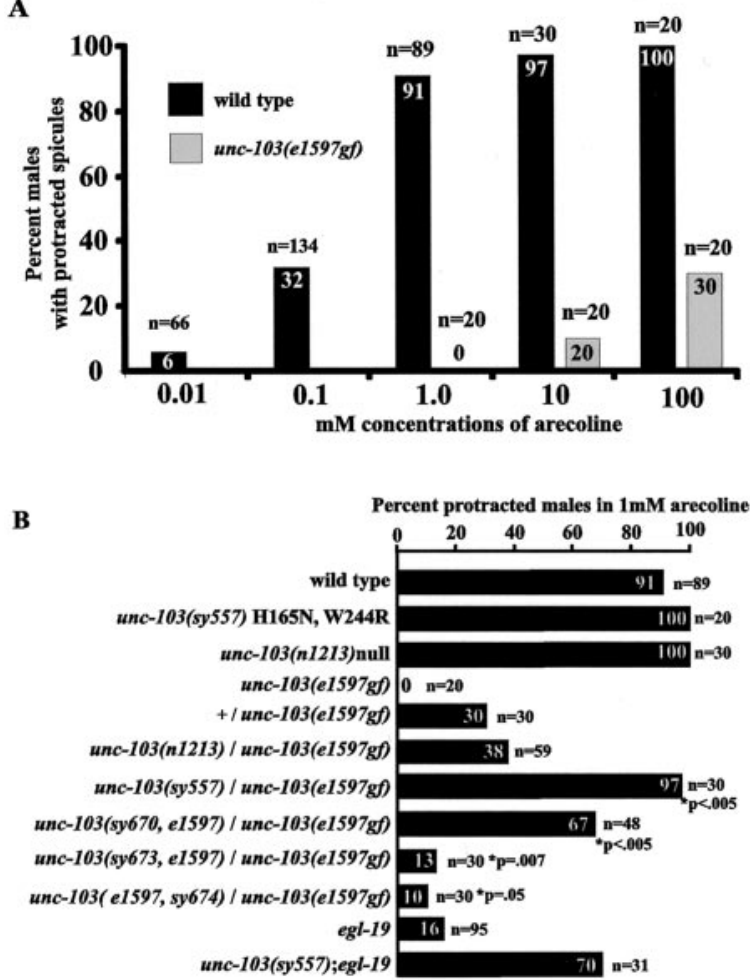

- Chl-squared test eompared to unc-103(n1213) / unc-103(e1597g

Figure 3. Arecoline-induced spicule protraction in unc- 103 mutants. $A$, The sensitivities of unc-103(e1597gf) males to different concentrations of arecoline are compared with wild type. The numbers in the columns refer to the percentages of males that protract their spicules with drug exposure; $n$ refers to the number of males assayed. $B$ shows how different unc-103 genotypes affect the percentages of males that protract their spicules in $1 \mathrm{~mm}$ arecoline.

cholinergic transmission from SPC, PCB, PCC, and additional neurons can cause the anal depressor and protractor muscles to contract (Garcia et al., 2001).

UNC-68 ryanodine receptor calcium channels are not essential for spicule insertion behavior, but they contribute to the frequency of periodic contractions. The unc-68(r1158) null allele reduced the unc-103(sy557)-induced protraction by one-half, suggesting that, in some animals, calcium efflux from sarcoplasmic stores can contribute to muscle seizures. However, because many unc-103(sy557);unc-68(r1158) males still had protracted spicules, intracellular calcium mobilization must not be essential.

Although mutations in $u n c-13$, unc-31, and unc-64 reduce synaptic transmission, they had little effect on the unc-103(sy557) phenotype. This finding was unexpected because reducing ACh production with the cha-1 mutation affected mating-independent spicule protraction. The unc-13, unc-31, and unc-64 alleles used in this work might not reduce synaptic transmission enough to affect spontaneous protraction drastically; alternatively, unc-103(sy557) might allow the neurons to bypass the requirement for wild-type action of these three genes.

unc-103 males execute premature prolonged protractor contraction during mating

When wild-type males mate with $24 \mathrm{hr}$ or younger adult hermaphrodites, they have difficulty inserting their spicules into the vulva. The tips of the spicules rapidly prod the vulval slit until eventually they penetrate the vulva. Prodding behavior is facilitated by UNC-68-mediated periodic protractor contractions. When the spicule tips penetrate the vulva, the SPC motor neu-
$\mathbf{A}$

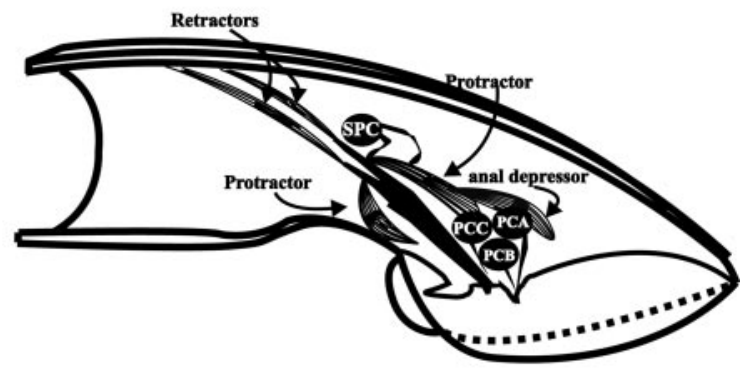

B

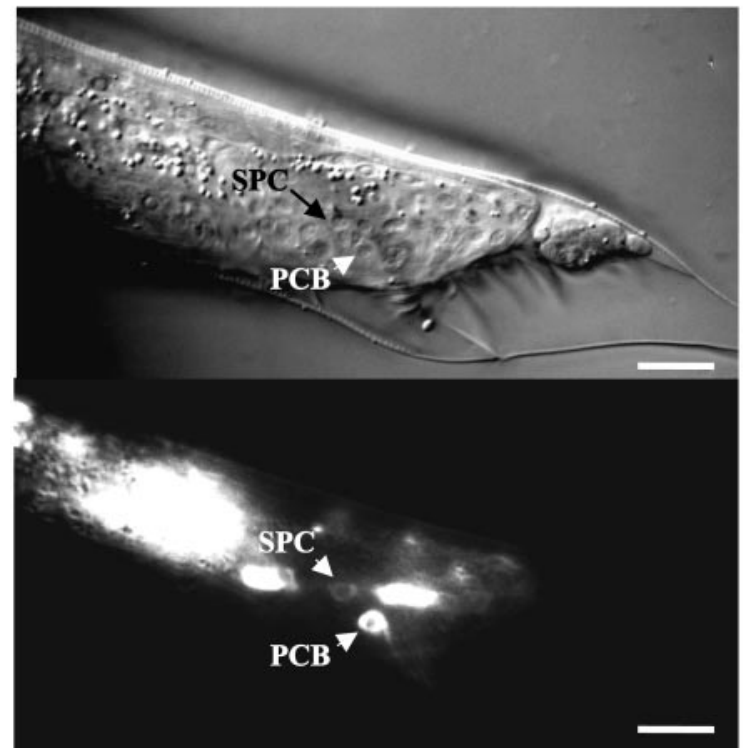

Figure 4. Cells involved in unc-103(sy557)-induced spicule protraction. $A$ depicts the right lateral view of the male tail, highlighting cells that are associated with the spicules. The drawing was adapted from Sulston et al. (1980). The spicule is shown in black. The dorsal and ventral protractor muscles, represented as striated cells, are attached to the body wall and the base of the spicule. The anal depressor muscle is attached to the body wall and contacts the dorsal protractor. The right SPC motor neuron contacts the protractors and the base of the spicule. The right $P C A, P C B$, and $P C C$ neurons send their sensory processes to the right postcloacal sensillum. $B$ depicts Nomarski (top) and fluorescence (bottom) images of the lateral tail region of a male that expresses the unc-103::GFP reporter construct. Scale bars, $20 \mu \mathrm{m}$. The male in the image is in $L 4$ lethargus. The PCB postcloacal sensory neuron and the SPC spicule motor neuron, in addition to some unidentified neurons, express the reporter gene.

rons have been proposed to trigger EGL-19-mediated prolonged contraction, causing the spicules to remain extended from the male tail (Garcia et al., 2001).

Although the unc-103 mutant phenotype suggests that UNC103 helps to keep the spicule muscles inactive before mating, the protein might function additionally during sex. To address this, we observed how nonprotracted $u n c-103$ males try to insert their spicules into $24 \mathrm{hr}$ adult hermaphrodites. We observed that, instead of consistently prodding the vulval slit with their spicule tips until penetration, all unc-103(sy557) H165N, W244R males $(n=2)$, unc-103(sy673, e1597gf) W85opal males $(n=10)$, and unc-103(n1213) null males $(n=10)$ repeatedly protract their spicules during prodding behavior. The prematurely extended spicules would depress the hermaphrodite vulva rather than penetrate it. After protraction the spicules retract into the male tail to 
Table 2. Ablations of protractor associated cells

\begin{tabular}{llcc}
\hline $\begin{array}{l}\text { Ablations in unc-103(sy557) } \\
\text { H165N, W244R }\end{array}$ & $\begin{array}{l}\text { Percentage of } \\
\text { protracted males }^{a}\end{array}$ & $n^{b}$ & $p^{b}$ value $^{e}$ \\
\hline Nonoperated & 59 & 75 & \\
P9.p, P10.p, P11.p & 83 & 12 & 0.11 \\
P(I/r), U(I/r) ${ }^{d}$ & 54 & 11 & 0.76 \\
PCA(I/r), PCB (I/r), PCC(I/r) & 88 & 8 & 0.12 \\
P9.p, P10.p, P11.p, PCA (I/r), & & & \\
$\quad$ PCB (I/r), anal depressor & 57 & 7 & 0.9 \\
SPD (1/r), SPV (1/r) & 50 & 8 & 0.6 \\
DVA, DVB, DVC & 43 & 14 & 0.25 \\
SPC(I/r) & 62 & 34 & 0.68 \\
Anal depressor & 63 & 19 & 0.76 \\
AVL, DVB, SPC (I/r) & 30 & 9 & 0.14 \\
SPC(I/r), anal depressor & 0 & 20 & $<0.005$ \\
\hline
\end{tabular}

${ }^{a}$ Males whose spicules remain permanently protracted.

${ }^{b}$ Number of males assayed.

'Ablations remove PGA, PDC, PVY, PVV, PVZ, HOA, HOB, and associated structural cells.

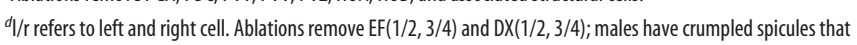
still protract.

${ }^{e} \chi^{2}$ test compared with nonoperated sy557.

Table 3. Mutant genes that affect unc-103(sy557)-induced protraction

\begin{tabular}{|c|c|c|c|}
\hline Genotype & $\begin{array}{l}\text { Percentage of } \\
\text { protracted males }^{b}\end{array}$ & $n^{c}$ & $p$ value ${ }^{d}$ \\
\hline Wild type $^{a}$ & 0 & 100 & \\
\hline unc-103(sy557) H165N, W244R ${ }^{1}$ & 66 & 454 & \\
\hline unc-38;unc-103(sy557) & 83 & 59 & n.d. \\
\hline unc-103(sy557);unc-49 & 72 & 53 & n.d. \\
\hline unc-103(sy557);unc-64 & 70 & 148 & n.d. \\
\hline unc-103(sy557);cat-1 & 60 & 27 & 0.2 \\
\hline unc-103(sy557);unc-2 & 54 & 28 & 0.18 \\
\hline unc-103(sy557);unc-31 & 52 & 85 & 0.02 \\
\hline unc-13; unc-103(sy557) & 47 & 43 & 0.02 \\
\hline unc-103(sy557);unc-68 & 31 & 45 & $<0.005$ \\
\hline unc-103(sy557);cha-1 & 11 & 63 & $<0.005$ \\
\hline unc-103(sy557);egl-19 & 5 & 60 & $<0.005$ \\
\hline
\end{tabular}

n.d, Not done.

${ }^{a}$ Values taken from Table 1.

${ }^{b}$ Males whose spicules remain permanently protracted.

Number of males assayed.

${ }^{d} \chi^{2}$ compared with sy557.

resume prodding behavior. We ablated the SPC motor neurons in five unc-103(n1213) null animals and observed their spicule insertion behavior. All of the operated males prematurely protracted their spicules during prodding behavior, demonstrating that the SPC motor neuron is not essential for this abnormal behavior.

We analyzed four intact unc-103(n1213) null males and four wild-type males in greater detail by counting the movements of their spicules. Video recordings of spicule insertion behavior were played back at slower speeds to determine the number of spicule movements during prodding behavior and the frequency of spontaneous protraction (Fig. 5). In contrast to the steady rhythmic prodding of wild-type spicules, we found that the spicules of unc-103(n1213) null males arrhythmically prodded the vulval slit 1-9 times/sec. We also noted that complete spicule protraction punctuated every $1-12 \mathrm{sec}$ of prodding behavior. Thus we conclude that unc-103 plays at least two roles in the male: to suppress protractor muscle contractions until proper mating signals are sensed and to regulate periodic and prolonged contractions during mating.

\section{Discussion}

UNC-103 keeps the spicule protractor muscles inactive before mating

Male mating behavior consists of many stereotyped subbehaviors that must be coordinated to insure efficient mating. To impregnate a hermaphrodite, the male moves backward along her body scanning for the vulva. If the male fails to locate the vulva on one side of the hermaphrodite, he will turn to her other side and resume vulva location behavior. When he locates her genitalia, he stops backward locomotion and adjusts the position of his tail over the vulva so that the spicule tips contact the vulval slit. The neurons used for vulva location behavior also signal the spicule protractor muscles to contract and relax rhythmically until the spicules penetrate the vulval barrier. When the spicules breach the vulva, the SPC motor neurons signal the protractor muscles to remain contracted until sperm transfer is completed.

To understand how different spicule muscle behaviors are regulated, we isolated mutations that cause males to protract their spicules in the absence of mating stimulation. From 10,000 mutagenized gametes we isolated unc-103(sy557). unc-103 (sy557) causes $\sim 60 \%$ of virgin males to protract their copulatory spicules within $24 \mathrm{hr}$ of adulthood. UNC-103 encodes a putative voltage-gated $\mathrm{K}^{+}$channel that is homologous to human $h$-erg and Drosophila seizure/erg. The sy557 lesion contains two mutations that create an $\mathrm{H} 165 \mathrm{~N}$ and W244R amino acid change in the $\mathrm{K}^{+}$channel protein. Null and loss-of-function alleles of $u n c-103$ also induce spontaneous spicule protraction, suggesting that before mating behavior UNC-103 keeps the spicule muscles from contracting. No other gross behavioral abnormalities are seen in unc-103(null) and unc-103(sy557) males, suggesting that cells in the spicule insertion behavioral circuit are more sensitive to changes in this $\mathrm{K}^{+}$channel than other unc-103-expressing cells.

\section{UNC-103 may act in parallel with other proteins to regulate protractor muscle activity}

Although unc-103(sy557) and the other unc-103 alleles cause mating-independent spicule protraction, males that do not protract their spicules within $24 \mathrm{hr}$ of adulthood will not display the abnormal phenotype later. Two scenarios can account for why some males do not show the defect: (1) buffering systems that act parallel to $u n c-103$ can overcompensate for loss of unc-103 function or (2) unaffected males are not exposed to some environmental stimuli that normally activates an unc-103-regulated excitatory pathway.

We favor the hypothesis that, at some efficiency, compensating mechanisms can attenuate spontaneous protractor activity in these exceptional males. The sy557 allele induces matingindependent spicule protraction at a higher percentage than compared with other $u n c-103$ alleles. This difference could be attributable to the sy557 protein interfering with these hypothetical compensating mechanisms. In our mutant screen we identified three additional mutations that cause premature spicule protraction similar to unc-103(sy557). These mutations are not in unc-103 and might affect components that act parallel to the $\mathrm{K}^{+}$ channel (our unpublished data).

Behavioral and pharmacological properties of unc-103(sy557)/+ and unc-103(sy557)/unc-103(gf) males suggest that unc-103 (sy557) encodes a dominant-negative protein that also may interfere promiscuously with other proteins. The H165N and W244R changes in the sy557 protein affect amino acids that are conserved in the human, fly, and worm protein. The hydrophobic aromatic to a hydrophilic basic side chain change at position 244 in the S5 transmembrane domain of $u n c-103$ may disrupt 


\section{A \\ unc-103 null}
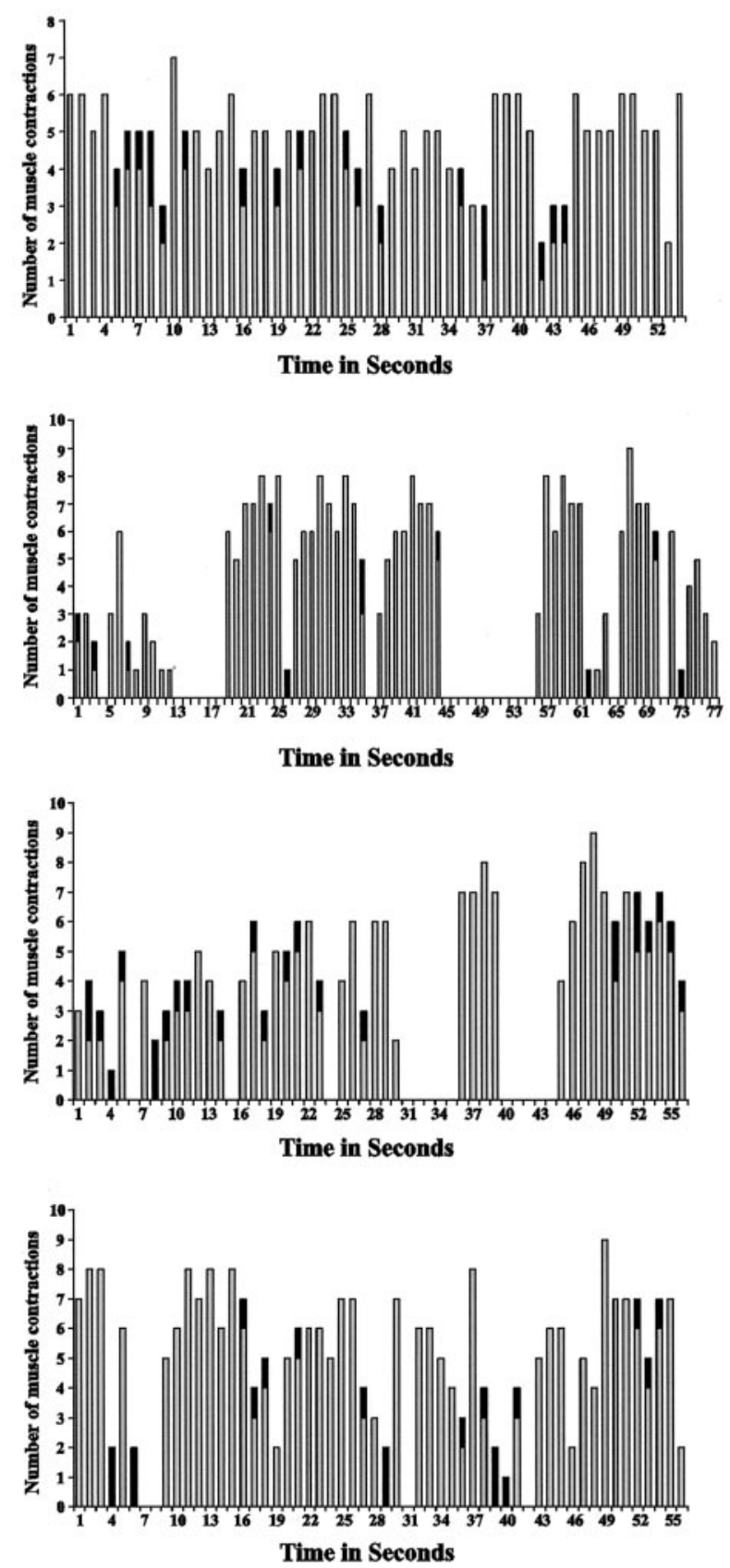

B wild type
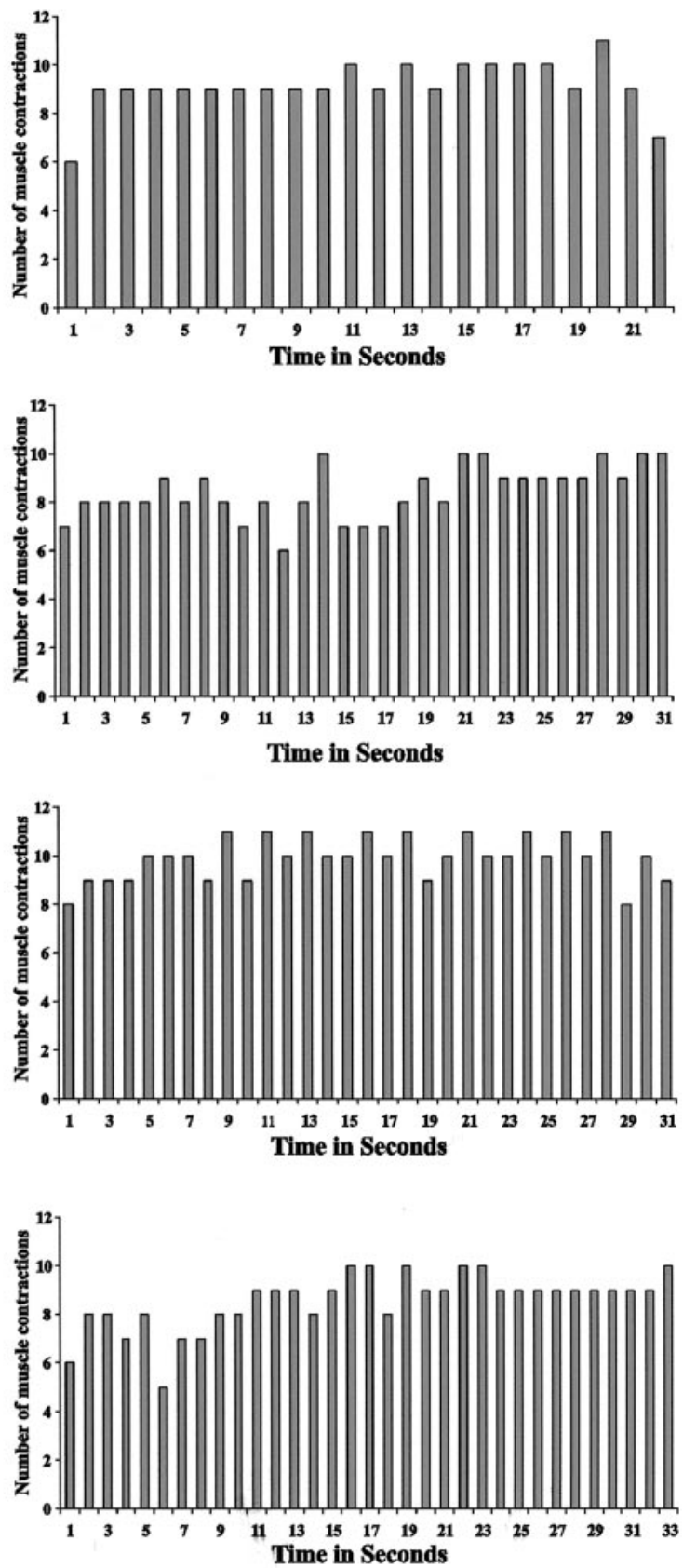

Figure 5. Number of muscle contractions per second during prodding behavior. A shows the profiles of muscle contractions of four unc-103(n1213) males. The horizontal axis displays sequential seconds during the window of observation. Gray columns represent the number of spicule movements during each second of prodding behavior. The black in some of the gray columns refers to a prolonged contraction that occurred during that time interval. Time intervals that show no gray columns are attributable to the hermaphrodites shifting their position during the observation period. Males temporarily stop prodding behavior to reorient their tails over the vulva. $B$ shows the profiles of muscle contractions of four wild-type males.

proper channel function. However, the consequence of the $\mathrm{H} 165 \mathrm{~N}$ substitution near the $\mathrm{S} 3$ transmembrane region is not obvious. UNC-103 shares $\sim 40 \%$ amino acid identity to the ether- $a$ go-go-encoded (eag) $\mathrm{K}^{+}$channels in humans, flies, and worms (Titus et al., 1997; Weinshenker et al., 1999). Interestingly, among the eag proteins asparagine is the conserved amino acid at that equivalent amino acid position near the $\mathrm{S} 3$ transmembrane region. We speculate that the sy557-encoded H165N substitution might cause unc-103 to interact with other $\mathrm{K}^{+}$channels artificially.
Neuronal ACh input facilitates unc-103(sy557)-induced spicule protraction

Ablation of both the SPC cholinergic neuron and the anal depressor muscle is required to suppress spontaneous spicule protraction. Removing either one separately has little effect, suggesting that both cells are capable of triggering the protractor muscles to sustain contraction. During mating behavior only the SPC motor neurons are essential for prolonged contraction. However, under artificial conditions neither SPC nor the anal depressor is re- 
quired because $\mathrm{ACh}$ agonists can activate protractor contraction directly even when both cell types are ablated (Garcia et al., 2001).

unc-103 is expressed in the SPC neurons, consistent with the contribution of those cells to the mutant phenotype. In hermaphrodites and larval males the AVL and DVB GABAergic neurons activate anal depressor muscle contractions (McIntire et al., 1993). In adult males these neurons might not contribute actively to unc-103(sy557)-induced protraction because killing these cells does not eliminate the mutant phenotype. The anal depressor muscle may have other inputs that facilitate spicule protraction in the adult male. We do not see unc-103 expression in the anal depressor, protractor, or any other type of muscle cell in the stable transgenic lines used in this study; however, because of caveats of using extrachromosomal arrays in C. elegans, we cannot rule out that unc-103 also might express in muscles (see Materials and Methods).

Similar to unc-103(sy557), aldicarb, an acetylcholine esterase inhibitor, can induce males to protract their spicules. Ablation of the SPC cholinergic motor neurons and the anal depressor muscle can attenuate aldicarb-induced protraction, demonstrating that $\mathrm{ACh}$ can act on or via these cells to induce the protractors to contract. Mutations in cha-1 choline acetyltransferase or unc-64 syntaxin also reduce aldicarb-induced protraction. Thus in the absence of mating stimulation low levels of cholinergic transmission to the protractor and anal depressor muscle occur (Garcia et al., 2001). In contrast to aldicarb-induced protraction, the unc103(sy557) phenotype is reduced by the cha-1 mutation, but not by an unc-64 mutation.

Worms containing unc-64 syntaxin mutations are severely compromised for locomotion. Elimination of SLO-1 calciumactivated, voltage-gated $\mathrm{K}^{+}$channels suppresses unc-64-induced lethargy. This observation suggests that unc-64 mutants express enough syntaxin proteins to promote locomotion, but not enough to overcome negative regulation of synaptic transmission by SLO-1 channels in ventral cord neurons (Wang et al., 2001). In cholinergic neurons that are involved specifically in spicule insertion behavior, UNC-103 in conjunction with SLO-1 and other $\mathrm{K}^{+}$channels might participate in attenuating UNC-64-mediated synaptic transmission. We speculate that unc-103(sy557) might suppress unc-64 synaptic transmission defects in mating-specific cells to induce spicule protraction.

\section{UNC-103 regulates prolonged contraction during mating}

During mating behavior ACh initiates two types of protractor muscle contractions that use different calcium channels. UNC-68 ryanodine receptor calcium channels are used during periodic contractions, and EGL-19 L-type voltage-gated calcium channels are needed for prolonged contraction. These calcium channels also are differentially required for levamisole-induced (an ACh agonist) and arecoline-induced spicule protraction. The difference between levamisole and arecoline activity is that the former requires UNC-68, whereas the latter requires EGL-19 (Garcia et al., 2001). We find that spicule protraction induced by unc103 (sy557) is similar to arecoline because egl-19 can suppress the unc-103(sy557) phenotype greater than unc-68. However, the unc-103 allele also partially can restore arecoline sensitivity to egl-19 males (Fig. 3B). We speculate that UNC-103 may regulate a signal transduction pathway that can be stimulated by arecoline, requires L-type voltage-gated calcium channels, and is used to prolong muscle contraction.

unc-103 mutants that do not protract their spicules permanently show a consistent abnormal spicule insertion behavior during mating. As unc-103 mutant males try to breach the vulva, the rhythm of periodic protractor contractions is abnormal and is disrupted by spurious prolonged contraction. In contrast to wild type, these premature prolonged contractions do not require the SPC motor neurons. Considering that the protractor muscles must distinguish between which types of cholinergic-induced contraction to use during spicule insertion behavior, UNC-103 might suppress EGL-19-used cholinergic pathways while the protractor muscles are undergoing $\mathrm{ACh}$-stimulated periodic contractions.

\section{References}

Ahmed S, Maruyama IN, Kozma R, Lee JH, Brenner S, Lim L (1992) The Caenorhabditis elegans unc-13 gene product is a phospholipid-dependent high-affinity phorbol ester receptor. Biochem J 287:995-999.

Alfonso A, Grundahl K, McManus JR, Rand JB (1994) Cloning and characterization of the choline acetyltransferase structural gene (cha-1) from C. elegans. J Neurosci 14:2290-2300.

Ann K, Kowalchyk JA, Loyet KM, Martin TF (1997) Novel $\mathrm{Ca}^{2+}$ binding protein (CAPS) related to UNC-31 required for $\mathrm{Ca}^{2+}$-activated exocytosis. J Biol Chem 272:19637-19640.

Atkinson NS, Robertson GA, Ganetzky B (1991) A component of calciumactivated potassium channels encoded by the Drosophila slo locus. Science 253:551-555.

Bamber BA, Beg AA, Twyman RE, Jorgensen EM (1999) The Caenorhabditis elegans unc-49 locus encodes multiple subunits of a heteromultimeric GABA receptor. J Neurosci 19:5348-5359.

Bargmann CI, Avery L (1995) Laser killing of cells in Caenorhabditis elegans. In: Methods of cell biology, Vol 48, Caenorhabditis elegans: modern biological analysis of an organism (Epstein HF, Shakes DC, eds), pp 225-250. New York: Academic.

Barker DM (1994) Copulatory plugs and paternity assurance in the nematode Caenorhabditis elegans. Anim Behav 48:147-156.

Barr MM, Sternberg PW (1999) A polycystic kidney-disease gene homologue required for male mating behaviour in C. elegans. Nature 401:386-389.

Brenner S (1974) The genetics of Caenorhabditis elegans. Genetics 77:71-94.

Cowan TM, Siegel RW (1986) Drosophila mutations that alter ionic conduction disrupt acquisition and retention of a conditioned odor avoidance response. J Neurogenet 3:187-201.

Curran ME, Splawski I, Timothy KW, Vincent GM, Green ED, Keating MT (1995) A molecular basis for cardiac arrhythmia: HERG mutations cause long QT syndrome. Cell 80:795-803.

Davis MW, Fleischhauer R, Dent JA, Joho RH, Avery L (1999) A mutation in the C. elegans EXP-2 potassium channel that alters feeding behavior. Science 286:2501-2504.

Doyle DA, Morais Cabral J, Pfuetzner RA, Kuo A, Gulbis JM, Cohen SL, Chait BT, MacKinnon R (1998) The structure of the potassium channel: molecular basis of $\mathrm{K}^{+}$conduction and selectivity. Science 280:69-77.

Duerr JS, Frisby DL, Gaskin J, Duke A, Asermely K, Huddleston D, Eiden LE, Rand JB (1999) The cat-1 gene of Caenorhabditis elegans encodes a vesicular monoamine transporter required for specific monoaminedependent behaviors. J Neurosci 19:72-84.

Elkes DA, Cardozo DL, Madison J, Kaplan JM (1997) EGL-36 shaw channels regulate C. elegans egg-laying muscle activity. Neuron 19:165-174.

Elkins T, Ganetzky B (1990) Conduction in the giant nerve fiber pathway in temperature-sensitive paralytic mutants of Drosophila. J Neurogenet 6:207-219.

Engel JE, Wu C-F (1998) Genetic dissection of functional contributions of specific potassium channel subunits in habituation of an escape circuit in Drosophila. J Neurosci 18:2254-2267.

Fleming JT, Squire MD, Barnes TM, Tornoe C, Matsuda K, Ahnn J, Fire A, Sulston JE, Barnard EA, Sattelle DB, Lewis JA (1997) Caenorhabditis elegans levamisole resistance genes $l e v-1, u n c-29$, and $u n c-38$ encode functional nicotinic acetylcholine receptor subunits. J Neurosci 17:5843-5857.

Garcia LR, Mehta P, Sternberg PW (2001) Regulation of distinct muscle behaviors controls the C. elegans male's copulatory spicules during mating. Cell 107:777-788.

Granato M, Schnabel H, Schnabel R (1994) pha-1, a selectable marker for gene transfer in C. elegans. Nucleic Acids Res 22:1762-1763.

Greenwald IS, Horvitz HR (1980) unc-93(e1500): a behavioral mutant of $C$. elegans that defines a gene with a wild-type null phenotype. Genetics 96:147-164.

Griffith LC, Wang J, Zhong Y, Wu CF, Greenspan R (1994) Calcium/ 
calmodulin-dependent protein kinase II and potassium channel subunit EAG similarly affect plasticity in Drosophila. Proc Natl Acad Sci USA 91:10044-10048.

Hodgkin J (1983) Male phenotypes and mating efficiency in Caenorhabditis elegans. Genetics 103:43-64.

Hodgkin JA, Doniach T (1997) Natural variation and copulatory plug formation in Caenorhabditis elegans. Genetics 146:149-164.

Hodgkin JA, Horvitz HR, Brenner S (1979) Nondisjunction mutants of the nematode Caenorhabditis elegans. Genetics 91:67-94.

Jackson FR, Wilson SD, Strichartz GR, Hall LM (1984) Two types of mutants affecting voltage-sensitive sodium channels in Drosophila melanogaster. Nature 308:189-191.

Jackson FR, Gitschier J, Strichartz GR, Hall LM (1985) Genetic modifications of voltage-sensitive sodium channels in Drosophila: gene dosage studies of the seizure locus. J Neurosci 5:1144-1151.

Johnstone DB, Wei A, Butler A, Salkoff L, Thomas JH (1997) Behavioral defects in C. elegans egl-36 mutants result from potassium channels shifted in voltage dependence of activation. Neuron 19:151-164.

Kamb A, Iverson LE, Tanouye MA (1987) Molecular characterization of Shaker, a Drosophila gene that encodes a potassium channel. Cell 50:405-413.

Kaplan WD, Trout WE (1969) The behavior of four neurological mutants of Drosophila. Genetics 61:399-409.

Kasbekar DP, Nelson JC, Hall LM (1987) Enhancer of seizure: a new genetic locus in Drosophila melanogaster defined by interactions with temperaturesensitive paralytic mutations. Genetics 116:423-431.

Kunkel MT, Johnstone DB, Thomas JH, Salkoff L (2000) Mutants of a temperature-sensitive two-P domain potassium channel. J Neurosci 20:7517-7524.

Lee RYN, Lobel L, Hengartner M, Horvitz HR, Avery L (1997) Mutations in the $\alpha 1$ subunit of an L-type voltage-activated $\mathrm{Ca}^{2+}$ channel cause myotonia in Caenorhabditis elegans. EMBO J 16:6066-6076.

Liu KS, Sternberg PW (1995) Sensory regulation of male mating behavior in Caenorhabditis elegans. Neuron 14:79-89.

Loer CM, Kenyon CJ (1993) Serotonin-deficient mutants and male mating behavior in the nematode Caenorhabditis elegans. J Neurosci 13:5407-5417.

Maryon EB, Coronado R, Anderson P (1996) unc-68 encodes a ryanodine receptor involved in regulating C. elegans body-wall muscle contraction. J Cell Biol 134:885-893.

McIntire SL, Jorgensen E, Kaplan J, Horvitz HR (1993) The GABAergic nervous system of Caenorhabditis elegans. Nature 364:337-341.

Mello CC, Kramer JM, Stinchcomb D, Ambros V (1991) Efficient gene transfer in C. elegans: extrachromosomal maintenance and integration of transforming sequences. EMBO J 10:3959-3970.

O'Dowd DK, Aldrich RW (1988) Voltage-clamp analysis of sodium channels in wild-type and mutant Drosophila neurons. J Neurosci 8:3633-3643.

Ogawa H, Harada S, Sassa T, Yamamoto H, Hosono R (1998) Functional properties of the unc-64 gene encoding a Caenorhabditis elegans syntaxin. J Biol Chem 273:2192-2198.

Papazian DM, Schwarz TL, Tempel BL, Timpe LC, Jan LY (1988) Ion channels in Drosophila. Annu Rev Physiol 50:379-394.

Park EC, Horvitz HR (1986) Mutations with dominant effects on the behavior and morphology of the nematode Caenorhabditis elegans. Genetics 113:821-852.

Pongs O, Kecskemethy N, Muller R, Krah-Jentgens I, Baumann A, Kiltz HH, Canal I, Llamazares S, Ferrus A (1988) Shaker encodes a family of putative potassium channel proteins in the nervous system of Drosophila. EMBO J 7:1087-1096.

Reiner DJ, Weinshenker D, Thomas JH (1995) Analysis of dominant muta- tions affecting muscle excitation in Caenorhabditis elegans. Genetics 141:961-976.

Reiner DJ, Newton EM, Tian H, Thomas JH (1999) Diverse behavioural defects caused by mutations in Caenorhabditis elegans unc-43 CaM kinase II. Nature 402:199-203.

Riddle DL (1977) A genetic pathway for dauer larva formation in C. elegans. Stadler Genet Symp 9:101-120.

Saifee O, Wei L, Nonet ML (1998) The Caenorhabditis elegans unc-64 locus encodes a syntaxin that interacts genetically with synaptobrevin. Mol Biol Cell 9:1235-1252.

Sanguinetti MC, Jiang C, Curran ME, Keating MT (1995) A mechanistic link between an inherited and an acquired cardiac arrhythmia: HERG encodes the $I_{\mathrm{Kr}}$ potassium channel. Cell 81:299-307.

Sanguinetti MC, Curran ME, Spector PS, Keating MT (1996) Spectrum of HERG K ${ }^{+}$channel dysfunction in an inherited cardiac arrhythmia. Proc Natl Acad Sci USA 93:2208-2212.

Schafer WR, Kenyon CJ (1995) A calcium-channel homologue required for adaptation to dopamine and serotonin in Caenorhabditis elegans. Nature 375:73-78.

Schnabel H, Schnabel R (1990) An organ-specific differentiation gene, pha-1, from Caenorhabditis elegans. Science 250:686-688.

Simon JM, Sternberg PW (2002) Evidence of a mate-finding cue in the hermaphrodite nematode Caenorhabditis elegans. Proc Natl Acad Sci USA 99:1598-1603.

Sulston J, Dew M, Brenner S (1975) Dopaminergic neurons in the nematode Caenorhabditis elegans. J Comp Neurol 163:215-226.

Sulston JE, Albertson DG, Thomson JN (1980) The Caenorhabditis elegans male: postembryonic development of nongonadal structures. Dev Biol 78:542-576.

Temple BL, Papazian DM, Schwarz TL, Jan YN, Jan LY (1987) Sequence of a probable potassium channel component encoded at the Shaker locus of Drosophila. Science 237:770-775.

Thomas JH (1990) Genetic analysis of defecation in Caenorhabditis elegans. Genetics 124:855-872.

Titus SA, Warmke JW, Ganetzky B (1997) The Drosophila erg K ${ }^{+}$channel polypeptide is encoded by the seizure locus. J Neurosci 17:875-881.

Trent C, Tsung N, Horvitz HR (1983) Egg-laying defective mutants of the nematode Caenorhabditis elegans. Genetics 104:619-647.

Trudeau MC, Warmke JW, Ganetzky B, Robertson GA (1995) HERG, a human inward rectifier in the voltage-gated potassium channel family. Science 269:92-95.

Wang XJ, Reynolds ER, Deak P, Hall LM (1997) The seizure locus encodes the Drosophila homolog of the HERG potassium channel. J Neurosci 17:882-890.

Wang ZW, Saifee O, Nonet ML, Salkoff L (2001) SLO-1 potassium channels control quantal content of neurotransmitter release at the C. elegans neuromuscular junction. Neuron 32:867-881.

Ward S, Carrel JS (1979) Fertilization and sperm competition in the nematode Caenorhabditis elegans. Dev Biol 73:304-321.

Waring DA, Kenyon CJ (1990) Selective silencing of cell communication influences anteroposterior pattern formation in C. elegans. Cell 60:123-131.

Warmke JW, Drysdale R, Ganetzky B (1991) A distinct potassium channel polypeptide encoded by the Drosophila eag locus. Science 252:1560-1562.

Weinshenker D, Wei A, Salkoff L, Thomas JH (1999) Block of an ether-ago-go-like $\mathrm{K}^{+}$channel by imipramine rescues egl-2 excitation defects in Caenorhabditis elegans. J Neurosci 19:9831-9840.

Wicks SR, Yeh RT, Gish WR, Waterston RH, Plasterk RHA (2001) Rapid gene mapping in Caenorhabditis elegans using a high density polymorphism map. Nat Genet 28:160-164. 